\title{
DERIVATIONS ON TERNARY RINGS OF OPERATORS
}

\author{
RoBERT Pluta AND BERNARD RUSSO
}

Abstract. To each projection $p$ in a $C^{*}$-algebra $A$ we associate a family of derivations on $A$, called $p$-derivations, and relate them to the space of triple derivations on $p A(1-p)$. We then show that every derivation on a ternary ring of operators is spatial and we investigate whether every such derivation on a weakly closed ternary ring of operators is inner.

Mathematics subject classification (2010): 47C15.

TRO.

Keywords and phrases: $\mathrm{C}^{*}$-algebra, ternary ring of operators, TRO, derivation, linking algebra, $\mathrm{W}^{*}$ -

\section{REFERENCES}

[1] T. J. Barton, Y. Friedman, Bounded derivations of JB*-triples, Quart. J. Math. Oxford Ser. (2) (1990), 255-268.

[2] C. J. K. BAtTY, Derivations of tensor products of $C^{*}$-algebras, J. London Math. Soc. (2) 17 (1978), $129-140$.

[3] ChO-Ho CHU, Jordan structures in geometry and analysis, Cambridge Tracts in Mathematics, vol. 190, Cambridge University Press, Cambridge, 2012.

[4] E. G. Effros, Z. J. RuAN, Operator Spaces, London Mathematical Society Monographs, vol. 23, Clarendon Press Oxford, 2000.

[5] M. Hamana, Triple envelopes and Silov boundaries of operator spaces, Math. J. Toyama Univ. bf 22 (1999), 77-93.

[6] J. Hamhalter, K. K. Kudaybergenov, A. M. Peralta, B. Russo, Boundedness of completely additive measures with application to 2-local triple derivations, J. Math. Physics 57 (2016), no. 2, 22 pp.

[7] T. Ho, J. Martínez-Moreno, A. M. Peralta, B. Russo, Derivations on real and complex JB* triples, J. London Math. Soc. (2) $\mathbf{6 5}$ (2002), no. 1, 85-102.

[8] T. Ho, A. M. PERALTA, B. RUSSO,Ternary weakly amenable $C^{*}$-algebras and JB*-triples, Quarterly J. Math. 64 (2013), 1109-1139.

[9] G. Horn, E. Neher, Classification of continuous JBW*-triples, Trans. Amer. Math. Soc. 306 (1988), 553-578.

[10] Manmohan KaUR, ZHONG-Jin RuAn, Local properties of ternary rings of operators and their linking $C^{*}$-algebras, J. Functional Analysis 195 (2002), 262-305.

[11] KuRT MEYBERG, Lectures on algebras and triple systems, The University of Virginia, Charlottesville, Va., 1972.

[12] Robert Pluta, Bernard Russo, Triple derivations on von Neumann algebras, Studia Math. 226 (2015), no. 1, 57-73.

[13] ZHONG-Jin RUAN, Type decomposition and the rectangular AFD property for $W^{*}-T R O s$, Canad. J. Math. 36 (2004), no. 4, 843-870.

[14] Bernard Russo, Derivations and projections on Jordan triples: an introduction to nonassociative algebra, continuous cohomology, and quantum functional analysis, In: Advanced courses of mathematical analysis V (2016), 118-227. World Sci. Publ., Hackensack, NJ.

[15] S. S AKAI, $C^{*}$-algebras and $W^{*}$-algebras, Ergebnisse der Mathematik und ihrer Grenzgebiete, vol. 60, Springer-Verlag, New York Heidelberg Berlin, 1971.

[16] A. M. Sinclair, Jordan homomorphisms and derivations on semisimple Banach algebras, Proc. Amer. Math. Soc. 24 (1970), 209-214. 
[17] W. Timmermann, Remarks on automorphism and derivation pairs in ternary rings of unbounded operators, Arch. Math. (Basel) 74 (2000), no. 5, 379-384.

[18] BORUT ZALAR, On the structure of automorphism and derivation pairs of $B^{*}$-triple systems, Topics in operator theory, operator algebras and applications (Timisoara 1994), Rom. Acad., Bucharest (1995), $265-271$. 